\title{
Lithium hydroxide dihydrate: A new type of icy material at elevated pressure
}

\author{
O. Tschauner, ${ }^{1,2, a)}$ B. Kiefer, ${ }^{3,4}$ M. Nicol, ${ }^{1}$ S. Sinogeikin, ${ }^{5}$ R. Kumar, ${ }^{1}$ and A. Cornelius ${ }^{1}$ \\ ${ }^{1}$ High Pressure Science and Engineering Center, Department of Physics, University of Nevada, Las Vegas, \\ Nevada 89154-4002, USA \\ ${ }^{2}$ Division of Geological and Planetary Sciences, California Institute of Technology, Pasadena, California \\ 91125, USA \\ ${ }^{3}$ Department of Physics, New Mexico State University, Las Cruces, New Mexico 88003, USA \\ ${ }^{4}$ Department of Chemical and Nuclear Engineering, University of New Mexico, Albuquerque, New Mexico \\ 87131-0001, USA \\ ${ }^{5}$ High Pressure Collaborative Access Team, Advanced Photon Source, Argonne National Laboratory, \\ Argonne, Illinois 60439, USA
}

(Received 19 August 2010; accepted 29 December 2010; published online 28 January 2011)

\begin{abstract}
We show that, in addition to the known monohydrate, $\mathrm{LiOH}$ forms a dihydrate at elevated pressure. The dihydrate involves a large number of H-bonds establishing chains along the $\langle 001\rangle$ direction. In addition, the energy surface exhibits a saddle point for proton locations along certain $\mathrm{O}$ interatomic distances, a feature characteristic for superprotonic conductors. However, MD simulations indicate that $\mathrm{LiOH} \cdot 2 \mathrm{H}_{2} \mathrm{O}$ is not a superprotonic conductor and suggest the relevant interpolyhedral $\mathrm{O}-\mathrm{O}$ distances being too large to allow for proton transfer between neighboring Li-coordinated polyhedra at least on the time scale of the MD-simulations. (c) 2011 American Institute of Physics. [doi:10.1063/1.3543797]
\end{abstract}

\section{INTRODUCTION}

Liquid $\mathrm{H}_{2} \mathrm{O}$ is a solvent for many polar compounds; however, at ambient pressure, water ice accepts only trace amounts of chemical impurities. ${ }^{1}$ However, at elevated pressure or at low temperatures, molecules or atoms of van der Waals radii much larger than that of $\mathrm{H}_{2} \mathrm{O}$ form hydrous compounds, where hydrogen-bonded ice networks encapsulate these molecules in large cages of so-called clathrate structures ${ }^{2,3}$ including clathrates of $\mathrm{H}_{2} \cdot{ }^{4-6}$

Recently, it was found that ice VII dissolves substantial amounts of $\mathrm{LiCl}^{7}$ These findings inspired us to search for light element hydrides forming clathrates or zeolitelike compounds with water ice.

Under ambient conditions, $\mathrm{LiH}$ reacts with water to form free, gaseous $\mathrm{H}_{2}$ and $\mathrm{LiOH}$. $\mathrm{LiOH}$ by itself can form a crystalline ionic monohydrate. ${ }^{8}$ While we observe that similar reactions prevail at higher pressures, at least up to $3.5 \mathrm{GPa}$, we find that $\mathrm{LiOH}$ forms a dihydrate at elevated pressure, which exhibits features characteristic for proton conductors: Li coordination polyhedra that can easily rotate and undersaturate coordinating oxygen atoms that can bind hydrogen.

\section{EXPERIMENTAL}

Single hexahedral crystals of LiH (Alfa) with $50 \mu \mathrm{m}^{3}$ edge lengths were loaded individually in the sample chambers of a diamond anvil cell. The chamber size was chosen such that half of the chamber was occupied by the hydride crystal. The chamber wall was established by a Re gasket of $100 \mu \mathrm{m}$

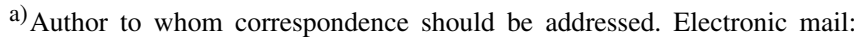
olivert@physics.unlv.edu.
}

thickness and with central bore of $150 \mu \mathrm{m}$ diameter and the culet of one diamond anvil. A spherule of annealed ruby of $5 \mu \mathrm{m}$ diameter was placed close to the inner border of the chamber. The second diamond anvil was then placed near the top of the gasket surface, leaving a gap of several $10 \mu \mathrm{m}$. Deionized water was injected with a syringe into this gap between anvil and gasket, while the whole assembly was observed under a microscope. Once water filled the chamber, the upper anvil was lowered within $1 \mathrm{~s}$ to close the chamber and to establish an initial pressure, which was clearly beyond the fluid $\mathrm{H}_{2}$-water coexistence boundary at $300 \mathrm{~K}$ since no gas formation was observed at this initial pressure. The mechanical load was raised subsequently to the experimental pressure. Three loadings were prepared at 1.5, 2.0, and 3.5 GPa.

All samples were examined by x-ray diffraction at the high flux microdiffraction undulator beamline ID-B at sector 16 (HPCAT) of the Advanced Photon Source (APS). A $34.325 \mathrm{keV}$-ray beam was focused in vertical and horizontal directions by Kirkpatrick-Baesz mirrors ${ }^{9}$ to a $5 \times 5 \mu \mathrm{m}^{2}$ spot at the sample chamber. The sample was rastered in a plane perpendicular to the beam direction over a $100 \times 100 \mu \mathrm{m}^{2}$ area during signal accumulation in order to maximize crystallite powder statistics. Diffracted radiation was recorded with an MAR345 image plate detector for average accumulation times of $300 \mathrm{~s}$ per frame. The recorded patterns were corrected for geometric distortion and integrated along the azimuthal angle. ${ }^{10}$ Instrumental profile parameters were extracted from Le Bail refinements ${ }^{11-13}$ of the patterns of an NIST $\mathrm{CeO}_{2}$ standard.

In all experiments we identified $\mathrm{LiOH} \cdot \mathrm{H}_{2} \mathrm{O}$, ice $\mathrm{VI}$, ice VII (at 2.0 and $3.5 \mathrm{GPa}$ ), and hydrogen-hydrates $\mathrm{C} 1$ (at the lowest pressure) and $\mathrm{C} 2 .{ }^{6}$ The presence of lithium hydroxide monohydrate and of hydrogen-clathrates in all three runs 


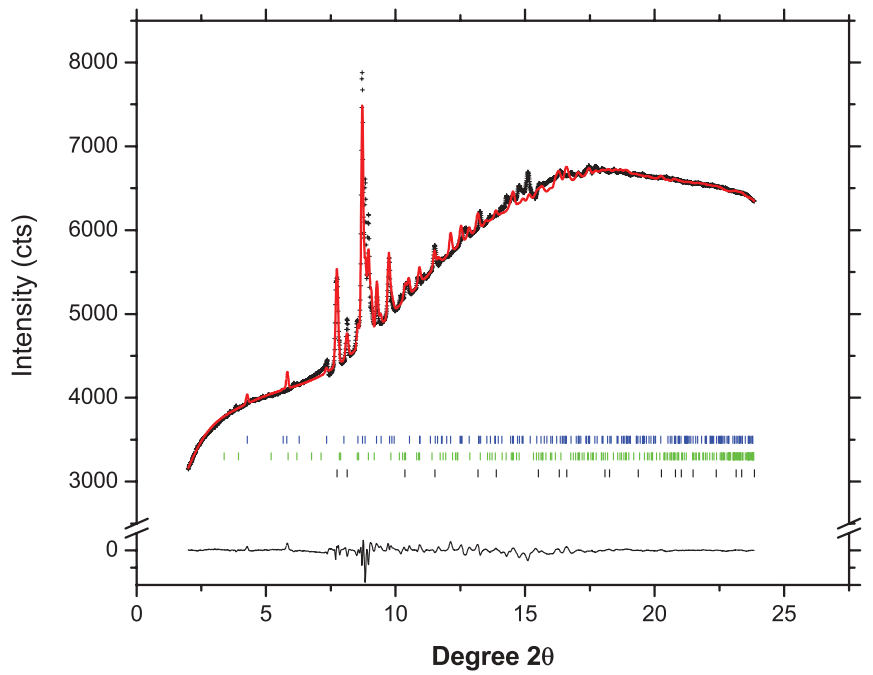

FIG. 1. Calculated (line) and observed (crosses) powder diffraction pattern of $\mathrm{LiOH} \cdot 2 \mathrm{H}_{2} \mathrm{O}$, phase $\mathrm{C} 2$, ice $\mathrm{VII}$ and $\mathrm{LiOH} \cdot \mathrm{H}_{2} \mathrm{O}$ at $3.5 \mathrm{GPa}$. Tickmarks indicate the $2 \vartheta$ angles of allowed reflections of $\mathrm{LiOH} \cdot 2 \mathrm{H}_{2} \mathrm{O}, \mathrm{C} 2$, and $\mathrm{LiOH} \cdot \mathrm{H}_{2} \mathrm{O}$, respectively. The bottom line represents the residual of calculated and observed pattern. All significant deviations (e.g. around $15^{\circ} 2 \vartheta$ ) are from misfit of the $\mathrm{C} 2$-phase (see experimental section for details).

indicates that $\mathrm{LiH}$ had reacted with water. Free hydrogen was captured in the $\mathrm{C} 1$ and $\mathrm{C} 2$ clathrates. No $\mathrm{LiH}$ was identified in any pattern.

In the run at $2.0 \mathrm{GPa}$, we identified ice VII, hydrogenhydrate $\mathrm{C} 1$, and $\mathrm{LiOH} \cdot \mathrm{H}_{2} \mathrm{O}$. In the run at $3.5 \mathrm{GPa}$, we found hydrogen-hydrate $\mathrm{C} 2$, ice $\mathrm{VII}, \mathrm{LiOH} \cdot \mathrm{H}_{2} \mathrm{O}$, and the diffraction pattern of an unknown phase. The pattern does not belong to any known phase of ice, Li-oxide, -hydroxide, or -hydride. The most intense diffraction features occur between $7.7^{\circ}$ and $10.3^{\circ} 2 \vartheta$, while there is a high density of reflections of low to moderate intensity at higher angles (Fig. 1) with increasing overlap at higher angles. As we will show, this new phase is $\mathrm{LiOH} \cdot 2 \mathrm{H}_{2} \mathrm{O}$.

The remaining large number of reflections indicates presence of a rather low symmetric structure or, alternatively, a mixture of unidentified phases. Besides the intrinsic difficulty of determining an unknown structure from powder data collected from a sample confined in a diamond anvil cell, we face the additional problem of unknown stoichiometry. We started to examine this unidentified part of the diffraction pattern by comparison to calculated patterns of known ice, clathrate, and zeolithe structures. We also calculated Li-bearing ice-network structures using known Li-silicate structures and substituting $\mathrm{Si}$ and $\mathrm{Al}$ by $\mathrm{O}$ and $\mathrm{O}$ by protons and subsequent compression of those model structures to densities comparable to $\mathrm{LiOH} \cdot \mathrm{H}_{2} \mathrm{O}$ or ice VII within $30 \%$ margins. This search showed similarity between the observed pattern and a calculated ice network derived from spodumene. ${ }^{14}$ Another approximate match was found for a layered structure of Li alternating with a two-dimensional ice network, which to our best knowledge has no immediate analog among known structures. We used these initial guesses as starting sets for reversed Monte Carlo (rMC) modeling using Free Objects for Crystallography (FOX). ${ }^{15}$ All rMC runs converged to one structural arrangement of atoms. Several indications support the conclusion that the result of the rMC modeling is not arbitrary: (a) the struc- ture has reasonable interatomic distances, (b) the same structure is found independently on the starting arrangement of atoms and even after reducing the initial set of reflections, (c) although the rMC calculations were run without symmetry constraints, the resulting structure has intrinsic symmetry (see below), (d) no bias by interatomic potentials had to be used, the resulting structure is robust with respect to the observed reflections, and finally, (e) additional rMC calculations using Endeavor 1.1 (Ref. 16) gave the same result.

An automatic symmetry search ${ }^{17}$ yielded monoclinic cells of space groups $\mathrm{C} 1 \mathrm{c} 1$ and $\mathrm{C} 12_{1} / \mathrm{m} 1$. However, the calculated pattern for the $\mathrm{C} 1 \mathrm{c} 1$ cell matched the observed pattern better.

\section{COMPUTATIONAL METHODOLOGY}

We augmented our experiments with first-principle density functional theory (DFT) calculations with two goals in mind: (1) investigate the stability of the structure obtained from x-ray diffraction and (2) to perform $a b$ initio molecular dynamics to investigate the likelihood of hydrogen mobility below the melting temperature of $\mathrm{LiOH} \cdot 2 \mathrm{H}_{2} \mathrm{O}$. All calculations were performed by using the software package VASP. ${ }^{18-21}$ An energy cutoff of $600 \mathrm{eV}$ was found sufficient to obtain converged results. The electronic band structure was determined using the tetrahedron method with Bloechl corrections. ${ }^{22}$ The interactions between ions and electrons were described within the projector-augmented-wave formalism; ${ }^{23,24}$ the reference configuration (core radii, in units of Bohr radii) were $1 \mathrm{~s}^{2} 2 \mathrm{~s}^{1}\left(2.050 \mathrm{a}_{\mathrm{B}}\right), 2 \mathrm{~s}^{2} 2 \mathrm{p}^{4}(1.520$ $\left.\mathrm{a}_{\mathrm{B}}\right)$, and $1 \mathrm{~s}^{1}\left(1.100 \mathrm{a}_{\mathrm{B}}\right)$, for $\mathrm{Li}, \mathrm{O}$, and $\mathrm{H}$, respectively. Electronic exchange and correlation effects were treated at the GGA level in the parametrization of Perdew et al. ${ }^{24}$ During the relaxation, all degrees of freedom, lattice and atomic positions, were relaxed simultaneously.

$A b$ initio MD simulations were performed for a 36 atom simulation cell of composition, $4 \mathrm{LiOH} \cdot 2 \mathrm{H}_{2} \mathrm{O}$. The cell shape of the DFT optimized static lattice. The temperature was controlled by a single Nose thermostat. ${ }^{25,26}$ The time step for the integration of Newton's equation of motion for the ions was 0.5 fs. The system evolution was followed up to 4000 timesteps ( 2 ps). We analyzed the time dependence of the mean-square displacement as a measure of mobility for that was analyzed for $\mathrm{Li}, \mathrm{O}$, and $\mathrm{H}$ separately. For a conventional superprotonic conductor it would be expected that the Li remains comparatively stationary, while the oxygen and the hydrogen subsystems should show larger excursions and occasional hydrogen jumps between $\mathrm{LiO}_{4}$ polyhedra. Thus, the mean-square displacement is a suitable measure for the assessment of mobility in superprotonic conductors. ${ }^{27}$

We compared the diffraction pattern of the relaxed DFT structures with the experimental diffraction pattern (Fig. 1). The experimental pattern exhibits spotty diffraction features from the comparatively coarse grained, coexisting monohydrate. These nonstatistical powder diffraction intensities affect the convergence of the Rietveld refinement. Therefore, we choose a different approach to compare experiment and theory, which bases on clearly identified reflections of the new phase only and which allows for removing reflections which 
TABLE I. List of observed and calculated structure factor moduli. First column: Miller indices of the observed reflections, second column: multiplicity, third column: observed structure factor moduli, and fourth column: calculated structure factor moduli based on the ab initio calculated model structure. The calculated moduli are corrected for multiplicity, while the observed moduli represent the combined values from contributions of overlapping reflections.

\begin{tabular}{|c|c|c|c|c|c|}
\hline \multicolumn{3}{|c|}{ hkl } & \multirow{2}{*}{$\frac{\text { Multiplicity }}{4}$} & \multirow{2}{*}{$\frac{\left|\mathrm{F}_{\mathrm{clc}}\right|}{4.33}$} & \multirow{2}{*}{$\frac{\left|\mathrm{F}_{\mathrm{obs}}\right|}{4.31}$} \\
\hline 1 & 1 & 0 & & & \\
\hline 1 & 1 & -1 & 4 & 1.82 & 3.641 \\
\hline 2 & 0 & 0 & 2 & 28.88 & 35.71 \\
\hline 0 & 2 & 0 & 2 & 5.67 & 3.60 \\
\hline 1 & 1 & 1 & 4 & 46.14 & 33.15 \\
\hline 0 & 2 & 1 & 4 & 4.67 & 8.03 \\
\hline 2 & 0 & -2 & 2 & 56.32 & 62.96 \\
\hline 3 & 1 & -1 & 4 & 45.94 & 45.65 \\
\hline 1 & 1 & -2 & 4 & 28.80 & 26.52 \\
\hline 2 & 2 & 0 & 4 & 37.38 & 32.69 \\
\hline 0 & 0 & 2 & 2 & 3.05 & 14.49 \\
\hline 3 & 1 & 0 & 4 & 15.10 & 18.10 \\
\hline 3 & 1 & -2 & 4 & 19.00 & 21.70 \\
\hline 2 & 2 & -2 & 4 & 37.38 & 32.69 \\
\hline 1 & 3 & -1 & 4 & 50.23 & 47.71 \\
\hline 4 & 0 & -2 & 2 & 4.58 & 1.58 \\
\hline 0 & 2 & 2 & 4 & 28.68 & 18.87 \\
\hline 2 & 2 & 1 & 4 & 7.41 & 6.03 \\
\hline 1 & 1 & 2 & 4 & 14.29 & 13.75 \\
\hline 1 & 3 & 1 & 4 & 5.2 & 9.65 \\
\hline 3 & 1 & -3 & 4 & 4.21 & 3.96 \\
\hline 4 & 2 & -2 & 4 & 18.97 & 29.73 \\
\hline 5 & 1 & -2 & 4 & 14.37 & 14.44 \\
\hline 2 & 4 & 0 & 4 & 26.93 & 22.10 \\
\hline 1 & 3 & 2 & 4 & 16.21 & 12.97 \\
\hline 4 & 2 & -3 & 4 & 27.56 & 29.16 \\
\hline 2 & 2 & 2 & 4 & 5.62 & 9.81 \\
\hline 5 & 1 & -3 & 4 & 18.67 & 24.37 \\
\hline 2 & 4 & -2 & 4 & 5.85 & 9.90 \\
\hline
\end{tabular}

are affected by contributions by overlapping Bragg peaks of coexisting phases: we extracted IF(hkl)l's by fitting peak profiles of the experimental pattern with the Le Bail method ${ }^{10}$ and compared them to the calculated structure factor moduli. Hereby, we did not partition $|\mathrm{F}(\mathrm{hkl})|$ 's of overlapping reflections but compared the observed $|\mathrm{F}(\mathrm{hkl})|$ 's to the calculated ones corrected for multiplicity. Up to a maximum $\mathrm{Q}$ of $5.6 \AA^{-1}$ sample reflections could be distinguished from reflections of the coexisting phases. We used the Wilson plot to identify and remove $\left|\mathrm{F}_{\text {obs }}\right|$ strongly affected by contributions from coexisting phases. The Wilson plot gives an average $\mathrm{B}_{\text {iso }}$ of $0.048(8)$. Out of 46 symmetry allowed reflections within a Q-range up to $5.6 \AA^{-1}$, there were 29 reflections of observable intensity, which did not substantially overlap with diffraction peaks from the coexisting phases and which could be used for evaluation of the structure model. The calculated and observed $|\mathrm{F}(\mathrm{hkl})|$ 's are listed in Table I.

Based on this set of reflections we calculate a reliability factor $\mathrm{R}=\Sigma\left(\left\|\mathrm{F}_{\mathrm{obs}}-\left|\mathrm{F}_{\mathrm{clc}} \| / \Sigma\right| \mathrm{F}_{\text {obs }} \mid\right)\right.$ of 0.17 , which indicates that the structure calculated by DFT represents the structure of the experimentally observed phase at least to the level of equal bond topology and coordination, while fractional coordinates may be different within the overall $17 \%$ margin of uncertainty given by the limited quality of the experimental data. However, comparison of the $a b$ initio calculated fractional coordinates with those obtained from the rMC modeling reveals markedly larger deviations for the $\mathrm{y}$-coordinates than for the $\mathrm{x}$ and $\mathrm{z}$ coordinates. This larger discrepancy could reflect an unfitted preferred orientation. More likely it is the result of omission of reflections due to overlap with the diffraction signal of the coexisting phases. In sum, the $\mathrm{R}_{\mathrm{int}}$ of $17 \%$ reflects an agreement between the experimental and $a b$ initio calculated fractional coordinates of $3 \%-15 \%$ for the $\mathrm{x}$ and $\mathrm{z}$ coordinates and only $30 \%$ for the $\mathrm{y}$-coordinate. Since the $a b$ initio calculation converged stably it is not likely that the y-coordinates of the atomic positions in the observed material deviate much more from the calculated values than the $\mathrm{x}$ and $\mathrm{z}$ coordinates. Fractional coordinates and cell parameters are given in Table I, and the structure model is shown in Figs. 2(a) and 2(b) in different projections. Upon compression or decompression, the quality of the diffraction patterns of lithium hydroxide dihydrate degrades significantly by peak broadening, which discouraged an examination of the structural evolution of this material with pressure.

\section{DISCUSSION}

We discuss $\mathrm{LiOH} \cdot 2 \mathrm{H}_{2} \mathrm{O}$ based on the structure calculated by DFT. All atoms reside on special positions. O1 and O2 have two protons each within $0.97-1.06 \AA$ distance. O3 has only one proton within $1.02 \AA$. Li has the closest distance to $\mathrm{O} 2(1.94 \AA)$, equal to the $\mathrm{Li}-\mathrm{O}$ distance in $\mathrm{LiOH} \cdot \mathrm{H}_{2} \mathrm{O}$ (Ref. 8), while all other O-atoms are not closer to $\mathrm{Li}$ than $2 \AA$. Clearly, $\mathrm{O} 1$ and $\mathrm{O} 2$ belong to water molecules, while $\mathrm{O} 3$ belongs to the hydroxide group.

$\mathrm{O} 1$ is tetrahedrally coordinated by H-bonds to adjacent $\mathrm{O} 1$ and $\mathrm{O} 2$ atoms (Fig. 1). These $\mathrm{H}$-bonds between $\mathrm{O} 1$ atoms form a chain along $\langle 100\rangle$ [Fig. 2(a)]. Another set of O-H... O interatomic distances involving $\mathrm{O} 2$ and $\mathrm{O} 3$ are longer than those expected for H-bonds (2.9-3.0 $\mathrm{A}$ ). If H-bonds would form along these directions $\mathrm{LiOH} \cdot 2 \mathrm{H}_{2} \mathrm{O}$ would be compatible with the Pauling rules of an ice-network forming channels along $\langle 010\rangle$. However, the static position of the proton attached to $\mathrm{O} 2$ is displaced from this potential bond direction along $\mathrm{O} 2-\mathrm{O} 3$, making the $\mathrm{O}-\mathrm{H}-\mathrm{H}$ distance markedly larger than expected for $\mathrm{H}$-bonds and distorting the hypothetical $\mathrm{H}-\mathrm{O}-\mathrm{H}$ bond angle substantially. This displacement is likely due to the influence of the electric field of $\mathrm{Li}$, which prohibits the formation of an ice network, at least at low temperatures. The $\mathrm{Li}-\mathrm{O} 3$ bond vectors and the $\mathrm{O} 3-\mathrm{H}$ bonds establish together a fivefold coordination of $\mathrm{O} 3$, while $\mathrm{Li}$ is threefold pyramidal coordinated by $\mathrm{O} 1, \mathrm{O} 2$, and $\mathrm{O} 3$ [Fig. 2(b)] considering $\mathrm{Li}-\mathrm{O}$ distances below $2.06 \AA$ (with a fourth distance at $2.33 \AA$ ). The projection in Fig. 2(b) shows how Li resides between the $\mathrm{H}_{2} \mathrm{O}$ tetrahedral "chains." It is remarkable that the distances between $\mathrm{Li}$ and the oxygen of the hydroxide on one side and the $\mathrm{O} 2$ of the water molecules on the other side differ by only $0.04 \AA$, making Li appearing like a "network former." This geometric configuration of $\mathrm{Li}-\mathrm{O}$ and $\mathrm{H}-\mathrm{O}$ bonds may permit for efficient proton conduction at elevated 


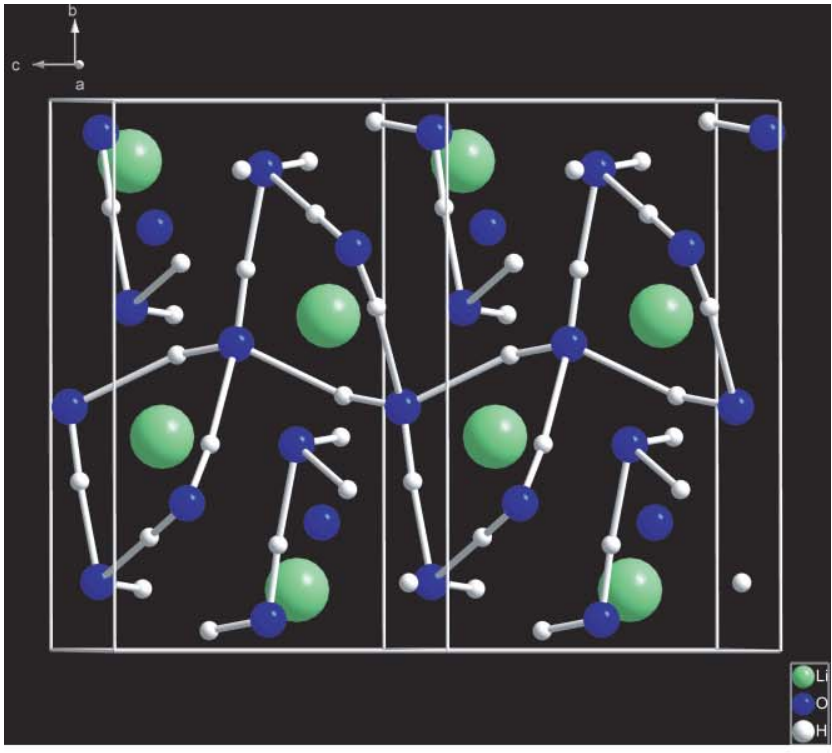

(a)

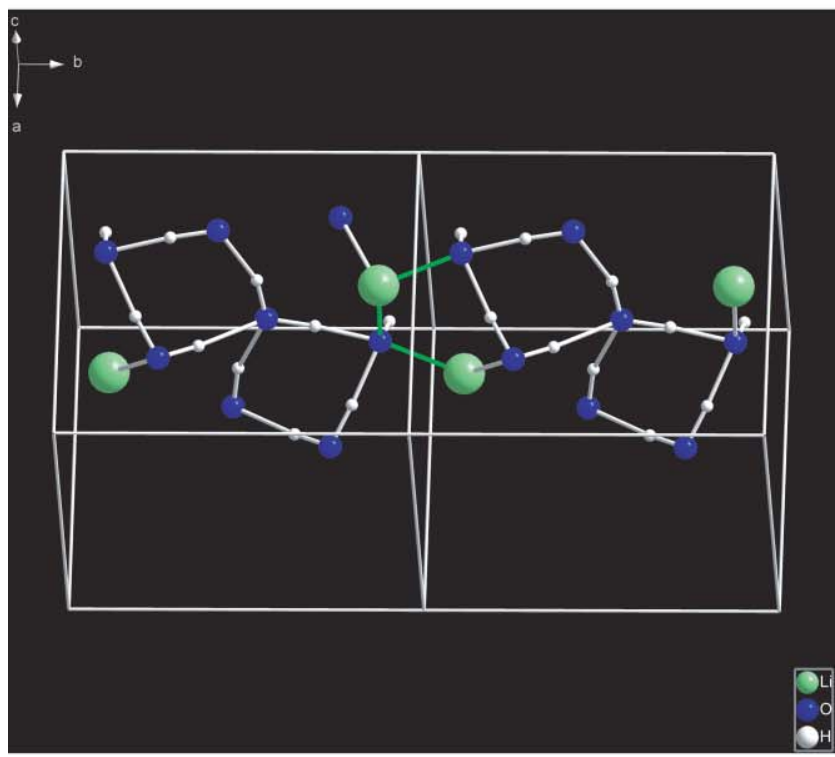

(b)

FIG. 2. (a) Representations of the structure of $\mathrm{LiOH} \cdot 2 \mathrm{H}_{2} \mathrm{O}$ as a ball-stick model. (a) Projection along $\langle 100\rangle$. Blue: $\mathrm{O}$, green: $\mathrm{Li}$, white: $\mathrm{H}$. White lines indicate the directions of $\mathrm{H}$-bonds. The most remarkable feature of the structure is the arrangement of water molecules along $\langle 100\rangle$ to a chain of fourmember rings. The lack of tetrahedral coordination of all oxygen atoms but $\mathrm{O} 1$ violates the ice-rules and prevents macroscopic disorder of protons. (b) Atom- and bond colors as above. Green lines: $\mathrm{Li}-\mathrm{O}$ distances below $2.0 \AA$, which are compatible with significant ionic interaction. The projection shows how $\mathrm{Li}$ resides between the $\mathrm{H}_{2} \mathrm{O}$ tetrahedral "chains." We note that the distances between $\mathrm{Li}$ and the oxygen of the hydroxide on one side and the $\mathrm{O} 2$ of the water molecules are shorter by only $0.04 \AA$, making $\mathrm{Li}$ appearing like a "network former." However, the MD simulations indicate that $\mathrm{LiOH} \cdot 2 \mathrm{H}_{2} \mathrm{O}$ does not behave like an itinerant ice network and does also not permit itinerant proton diffusion paths which would make this material a superb proton conductor.

temperature, if correlated $\mathrm{Li}$ - and $\mathrm{O}$-motion serves as a mediator for itinerant proton diffusion paths.

Elevated temperature in combination with an applied electric potential could make $\mathrm{LiOH} \cdot 2 \mathrm{H}_{2} \mathrm{O}$ a proton conductor of very high proton density but rather low bulk density.
TABLE II. Atomic positions for $\mathrm{LiOH} \cdot 2 \mathrm{H}_{2} \mathrm{O}$ calculated $a b$ initio at $9.5 \mathrm{GPa}$ and $0 \mathrm{~K}$. The calculated cell parameters are $\mathrm{a}=6.3714 \AA$, $\mathrm{b}=7.6916 \AA, \mathrm{c}=5.0619 \AA$, and $\beta=121.369^{\circ}$. The experimental cell parameters at $3.5 \mathrm{GPa}$ were found to be $\mathrm{a}=7.697(1) \AA, \mathrm{b}=6.624(7) \AA$, $\mathrm{c}=4.518(4) \AA$, and $\beta=112.17(9)^{\circ}$. The space group is $\mathrm{C} 1 \mathrm{c} 1$ (\#9).

\begin{tabular}{lcccc}
\hline \hline & Wyckoff & $x$ & $y$ & $z$ \\
\hline $\mathrm{Li}$ & $4 \mathrm{a}$ & 0.3661 & 0.1099 & 0.3309 \\
$\mathrm{O} 1$ & $4 \mathrm{a}$ & 0.2754 & 0.5574 & 0.4952 \\
$\mathrm{O} 2$ & $4 \mathrm{a}$ & 0.0399 & 0.2318 & 0.1938 \\
$\mathrm{O} 3$ & $4 \mathrm{a}$ & 0.3026 & 0.1246 & 0.9144 \\
$\mathrm{H} 1$ & $4 \mathrm{a}$ & 0.9421 & 0.1247 & 0.1055 \\
$\mathrm{H} 2$ & $4 \mathrm{a}$ & 0.2837 & 0.3069 & 0.9673 \\
$\mathrm{H} 3$ & $4 \mathrm{a}$ & 0.2467 & 0.5370 & 0.6673 \\
$\mathrm{H} 4$ & $4 \mathrm{a}$ & 0.1303 & 0.1112 & 0.7500 \\
$\mathrm{H} 5$ & $4 \mathrm{a}$ & 0.9531 & 07059 & 0.7908 \\
\hline \hline
\end{tabular}

Based on the mean-square displacements, the molecular dynamic calculation at $1000 \mathrm{~K}$ indicates that all atomic species move. The material is therefore likely molten. At 500 and $750 \mathrm{~K}$ we find no translational motion of any component and atoms move about their equilibrium positions. In particular, none of the hydrogen atoms moves within a 2 ps time period. This indicates that there is no indication of proton conduction, at least on the time scale of the simulations. The time scale of $\sim 1$ ps has been observed previously in superprotonic conductors as a typical time scale associated with the hydrogen hopping. ${ }^{22,27-29}$ The time averaged structure of $\mathrm{LiOH} \cdot 2 \mathrm{H}_{2} \mathrm{O}$ at $500 \mathrm{~K}$ is consistent with the (initial) static structure (Table II): all atoms including the hydrogens are within $\sim 0.04 \AA$. The structure of $\mathrm{LiOH} \cdot 2 \mathrm{H}_{2} \mathrm{O}$ appears to be stable at $500 \mathrm{~K}: \sim 50 \%$ of the static internal coordinates are within one standard deviation of each other and the free enthalpies of the $a b$ initio and the MD-calculated phase agree to within $\sim 0.6 \mathrm{meV} /$ atom.

The absence of proton diffusion in these simulations indicates that the $\mathrm{O} 2-\mathrm{H}-\mathrm{O} 3$ distance vectors do not correspond to easy diffusion paths even at elevated temperature. Thus, an itinerant network of proton paths throughout the lattice does not form. Moreover, the simulations indicate that the protons attached to the $\mathrm{O} 1$ and $\mathrm{O} 2$ atoms do not translate. This negative result can be tentatively assigned to the poorly screened nuclear charge of the Li ions: the oxygen ions are more strongly bound, and consequently, the potential wells for the hydrogen are deeper which makes proton hopping a less likely event. The rather short $\mathrm{Li}-\mathrm{O} 2$ distance of $1.98 \AA$ is consistent with such a strong influence of the ionic charge of $\mathrm{Li}$ and subsequent confinement of the protons attached to $\mathrm{O} 2$.

\section{CONCLUSIONS}

We report a new dihydrate of $\mathrm{LiOH}$ to form at elevated pressure. The structure of this material was determined by comparing the $a b$ initio calculated structure with the findings from experimental synchrotron x-ray diffraction patterns, where calculated and observed structure factors agree with an $R_{\text {int }}$ of 0.17 . The structure exhibits an unusual topology of chains of H-bonds along $\langle 100\rangle$. Together with a second set of 
O-H... O interatomic distances, oxygen and protons establish an array of Li-filled channels along $\langle 101\rangle$ compatible with the Pauling rules of an ice network However, the $\mathrm{O}-\mathrm{H}$ distances of this second set are too long and the $\mathrm{O}-\mathrm{H}_{4}$ tetrahedra too distorted to establish H-bonds. We examined the possibility that elevated temperature may restore proton diffusion along those distance vectors. This would make $\mathrm{LiOH} \cdot 2 \mathrm{H}_{2} \mathrm{O}$ a strong and lightweight candidate for proton conduction. However, our MD simulations do not show selective hydrogen motion. That is either all atoms show excursions about their equilibrium positions or all atoms move simultaneously, indicating that the material is in a liquid state. The disruption of the $\mathrm{H}$-network and the deep potential energy well for the protons both by the poorly screened charge field of Li are likely reasons for the absence of significant proton conduction in this material.

\section{ACKNOWLEDGMENTS}

This work was supported through the NNSA Cooperative Agreement DE-FC52-06NA27684 and Department of Energy (DOE) Award DE-FG36-05GO08502 for hydrogen fuel cells and storage technology. Use of the HPCAT facility was supported by DOE-BES, DOE-NNSA, National Science Foundation (NSF), DOD-TACOM, and the W.M. Keck Foundation. APS is supported by DOE-BES under Contract No. W-31-109-Eng-38.

${ }^{1}$ V. F. Petrenko and R. W. Whitworth, Physics of Ice (Oxford University Press, Oxford, 1999).

${ }^{2}$ J. S. Loveday and R. J. Nelmes, Chem. Phys. 10, 937 (2008).

${ }^{3}$ B. C. Chakoumakos, Am. Mineral. 89, 1153 (2004).
${ }^{4}$ W. L. Mao, H. K. Mao, A. F. Goncharov, V. V. Struzhkin, Q. Z. Guo, J. Z. $\mathrm{Hu}$ J. F. Shu, R. J. Hemley, M. Somayazulu, and Y. S. Zhao, Science 297, 2247 (2002).

${ }^{5}$ S. Patchkovskii and J. S. Tse, Proc. Natl. Acad. Sci. U.S.A. 100, 14645 (2003)

${ }^{6}$ W. L. Vos, L. S. Finger, R. J. Hemley, and H. K. Mao, Phys. Rev. Lett. 71, 3150 (1993).

${ }^{7}$ S. Klotz, L. E. Bove, T. Straessle, T. C.Hansen, and A. M. Saitta, Nature Mater. 8, 405 (2009)

${ }^{8}$ N. W. Alcock, Acta Cryst. B 27, 1682 (1971).

${ }^{9}$ B. X. Yang, M. Rivers, W. Schildkamp, and P. J. Eng, Rev. Sci. Instrum. 66, 2278 (1995).

${ }^{10}$ A. P. Hammersley, S. O. Svensson, M. Hanfland, A. N. Fitch, and D. Häusermann, High Press. Res. 14, 235 (1996).

${ }^{11}$ A. Le Bail, H. Duroy, and J. L. Fourquet, Math Res. Bull. 23, 447 (1988).

${ }^{12}$ A. C. Larson and R. B. Von Dreele, Los Alamos National Laboratory Report LAUR 86, 1994.

${ }^{13}$ N. W. Alcock, Acta Cryst. B 27, 1682 (1971)

${ }^{14}$ M. Cameron, S. Sueno, C. T. Prewitt, and J. J. Papike, Am. Mineral. 58, 594 (1973).

${ }^{15}$ V. Favre-Nicolin and R. Cerny, J. Appl. Crystallogr. 35, 734 (2002).

${ }^{16}$ H. Putz, J. C. Schön, and M. Jansen, J. Appl. Crystallogr. 32, 864 (1999).

${ }^{17}$ A. L. Speck, PLATON, a multipurpose crystallographic tool Utrecht University, Utrecht, The Netherlands, 2001. See http://www.cryst.chem. uu.nl/platon/.

${ }^{18}$ G. Kresse and J. Furthmuller, Comput. Mater. Sci. 6, 15 (1996).

${ }^{19}$ G. Kresse and J. Hafner, J. Phys.: Condens. Matter. 6, 8245 (1994).

${ }^{20}$ G. Kresse and J. Furthmuller, Phys. Rev. B 54, 11169 (1996).

${ }^{21}$ P. E. Blöchl, O. Jepsen, and O. K. Andersen, Phys. Rev. B 49, 16223 (1994).

${ }^{22}$ P. E. Blöchl, Phys. Rev. B 50, 17953 (1994).

${ }^{23}$ G. Kresse and D. Joubert, Phys. Rev. B 59, 1758 (1999).

${ }^{24}$ J. P. Perdew, K. Burke, and M. Ernzerhof, Phys. Rev. Lett. 77, 3865 (1996).

${ }^{25}$ S. Nosé, J. Chem. Phys. 81, 511 (1984).

${ }^{26}$ S. Nosé, Prog. Theor. Phys. Suppl. 103, 1 (1991).

${ }^{27}$ H.-S. Lee and M. E. Tuckerman, J. Phys. Chem. C 112, 9917 (2008).

${ }^{28}$ C. R. I. Chisholm and Y. H. Jang, Phys. Rev. B 72, 134103 (2005).

${ }^{29}$ B. C. Wood and N. Marzari, Phys. Rev. B 76, 134301 (2007). 